\title{
Laserspray Ionization (LSI) Ion Mobility Spectrometry (IMS) Mass Spectrometry
}

\author{
Ellen Inutan and Sarah Trimpin \\ Department of Chemistry, Wayne State University, Detroit, Michigan, USA
}

A simple device is described for desolvation of highly charged matrix/analyte clusters produced by laser ablation leading to multiply charged ions that are analyzed by ion mobility spectrometry-mass spectrometry. Thus, for example, highly charged ions of ubiquitin and lysozyme are cleanly separated in the gas phase according to size and mass (shape and molecular weight) as well as charge using Tri-Wave ion mobility technology coupled to mass spectrometry. This contribution confirms the mechanistic argument that desolvation is necessary to produce multiply charged matrix-assisted laser desorption/ionization (MALDI) ions and points to how these ions can be routinely formed on any atmospheric pressure mass spectrometer. (J Am Soc Mass Spectrom 2010, 21, 1260-1264) (c) 2010 American Society for Mass Spectrometry

$\mathrm{L}$ aserspray ionization (LSI) mass spectrometry (MS) was recently introduced on a Thermo Fisher Scientific Orbitrap Exactive [1-3]. The principle of this ionization method is that the analyte/matrix sample is ablated by the use of a laser operating at atmospheric pressure (AP) and ions are subsequently formed from highly charged matrix/analyte clusters during a desolvation process. The free choice of charge state selection demonstrates the utility of LSI for the analysis of complex mixtures. Singly charged ions similar to those obtained with matrix-assisted laser desorption/ ionization (MALDI) or multiply charged ions similar to those produced by electrospray ionization (ESI) [2] can be selected using LSI. Multiply charged ions are especially beneficial for providing the ability to ionize by laser ablation larger molecules such as proteins and synthetic polymers on high-performance but mass range limited mass spectrometers such as the Orbitrap Exactive [2,3]. Full range mass spectra of bovine insulin were obtained on as little as $40 \mathrm{fmol}$ when applying ion transfer capillary temperatures of $\sim 350{ }^{\circ} \mathrm{C}$ [3].

The unique feature of LSI to produce highly charged ions using direct laser ablation of a solid surface enhances fragmentation as was demonstrated by obtaining nearly complete protein sequence coverage of ubiquitin using electron-transfer dissociation (ETD) technology on a LTQ mass spectrometer [3]. Initial laserspray applications demonstrated the ability to produce singly charged lipid ions from mouse brain tissue under ambient conditions [1]. Using transmission geometry the matrix treated tissue sections were passed free-hand through the focused laser beam in front of the mass spectrometer orifice (Orbitrap

Address reprint requests to Dr. S. Trimpin, Department of Chemistry, Wayne State University, 5101 Cass Ave., Detroit, MI 48202, USA. E-mail: strimpin@chem.wayne.edu
Exactive) obtaining a spatial resolution of $<100 \mu \mathrm{m}$ [1]. The objective is to observe proteins from tissue.

Ion mobility spectrometry (IMS) MS has many advantages compared with even high-resolution mass spectrometers because of its ability to extend the dynamic range and separate isomeric composition [4-7]. This is possible because ions are separated in the IMS dimension according to charge and cross-section (size and shape) [8-11]. One key benefit of this solvent-free gas-phase separation by IMS is that when combined with solvent-free sample preparation [12] achieves total solvent-free analysis by MS entirely decoupling ionization, separation, and mass analyses from the use of any solvent [13-15].

We rationalized that to produce LSI multiply charged ions from the proposed highly charged matrix/analyte clusters [3], a device is required that efficiently desolvates the 2,5-dihydroxybenzoic acid (2,5-DHB) matrix to produce the highly charged molecular ions. One function of the Waters Company (Manchester, UK) ESI z-spray source design used with the IMS-MS SYNAPT G2 is to reduce the background arising from cluster ions. However, this arrangement is not satisfactory as a means of desolvating clusters in which the matrix (serving as solvent) is 2,5-DHB. We, therefore, fabricated a simple device that is mounted on the z-spray ESI source and provides the thermal conditions necessary for desolvation and multiply charged ion production using the LSI method. The benefits of IMS-MS in combination with direct LSI ambient ionization for the efficient separation and characterization of a protein mixture is demonstrated.

\section{Experimental}

\section{Materials}

The 2,5-dihydroxybenzoic acid (DHB) matrix (98\% purity), bovine insulin, ubiquitin (bovine erythrocytes), and lysozyme (chicken eggwhite) were purchased from 
Sigma Aldrich, Inc., St. Louis, MO, USA, and used as is. Acetonitrile $(\mathrm{ACN})$ and methanol $(\mathrm{MeOH})$ solvents were obtained from Fisher Scientific Inc., Pittsburgh, PA, USA. Purified water was used (Millipore Corp., Billerica, MA, USA). Microscopy slides (dimensions $1 \times$ 3 in.) were obtained from Gold Seal Products, Portsmouth, NH, USA.

\section{Fabrication of the Temperature Device}

The fabricated external device for producing a relatively constant temperature is shown in the Graphical Abstract. This device is mounted externally to the z-spray ESI skimmer of the SYNAPT G2 IMS-MS mass spectrometer. Copper tubing (1/8 in. o.d., $1 / 16$ in. i.d. $\times$ 1 in. L), was coated with a layer of Sauereisen cement (Inso-lute Adhesive Cement Powder no. P1) wrapped with 24 gauge nichrome wire (Science Kit and Boreal Laboratories, Division of Science Kit, Inc., Tonawanda, NY, USA) and finally coated with another layer of Sauereisen cement. One end of the copper tubing was sanded to fit through the cover desolvation device (cone gas nozzle) and over the end of the ion entrance skimmer (sample cone). The copper tube was held to the cone gas nozzle with Sauereisen cement. Both ends of the coiled nichrome wire were connected with alligator clips to the insulated copper wire from a variac (Powerstat Variable Transformer type 116). The copper desolvation device was heated by application of up to $12 \mathrm{~V}$ from the variac. The temperature measured on the outside of the device was $>200{ }^{\circ} \mathrm{C}$.

\section{Sample Preparation}

Stock solutions of ubiquitin (117 pmol $\left.\mu \mathrm{L}^{-1}\right)$ and lysozymes (70 pmol $\mu \mathrm{L}^{-1}$ ) were prepared individually in pure water. Bovine insulin was dissolved in 50:50 $\mathrm{MeOH}: \mathrm{H}_{2} \mathrm{O}$ to give a 50 pmol $\mu \mathrm{L}^{-1}$ stock solution. For the protein mixture, ubiquitin and lysozyme were premixed using the stock solution in a 1:1 volume ratio and $1 \mu \mathrm{L}$ was used to prepare the LSI sample on the microscope slide employing solvent-based sample preparation protocols using 2,5-DHB matrix $[16,17]$ and prepared in 50:50 ACN: $\mathrm{H}_{2} \mathrm{O}$ and then dried to completeness. The dried LSI sample was placed in front of the desolvation device in a distance of about 1 to $3 \mathrm{~mm}$, similar to studies in which the dried sample was placed directly in front of the mass spectrometer orifice of the Orbitrap Excative [1-3].

\section{Laserspray Ionization}

The principle of LSI-MS is shown in the Graphical Abstract and has been described in greater detail previously [1-3]. The source configuration of the Nanolockspray ion source not only needs desolvation modifications but changes to the focusing alignment. This was achieved by removing the lockspray motor of the Nanolockspray ion source. A nitrogen laser (Spectra Physics VSL-
337ND-S, Mountain View, CA, USA) was mounted so that the laser beam fires at a $180^{\circ}$ angle (transmission geometry) directly at the ion entrance orifice of the skimmer of the SYNAPT G2 mass spectrometer. The $\mathrm{x}, \mathrm{y}, \mathrm{z}$-stage of the Nanolockspray ion source was used to move the glass microscope slide used as a LSI sample holder relative to the copper tube. The LSI sample holder with matrix/analyte applied was slowly moved through the focused laser beam.

\section{Ion Mobility Spectrometry-Mass Spectrometry}

The SYNAPT G2 HDMS was used to perform LSIIMS-MS analysis; a detailed description of the first generation instrument has been previously provided [18]. The scan time was set for $1 \mathrm{~s}$. All acquisitions were obtained for 2 min although sampling of the spot was frequently completed in a shorter time period. The gas used for the drift time $\left(t_{d}\right)$ separation was nitrogen with a flow set at $22 \mathrm{~mL} \mathrm{~min}^{-1}$. The pressure in the drift cell of $3.29 \mathrm{mBar}$ was recorded. A wave velocity was set to $300 \mathrm{~m} \mathrm{~s}^{-1}$. The IMS wave height was set from $8 \mathrm{~V}$ to 20 V. After acquisition, the data were evaluated using DriftScope ver. 2.1 (Waters Corp., Manchester, UK) where 2-D plots of $\boldsymbol{t}_{\boldsymbol{d}}$ versus mass-to-charge $(\mathrm{m} / \mathrm{z})$ ratio separation can be visualized. For the display of the 2-D plots, white background and inverse gray scale was used as DriftScope settings.

\section{Results and Discussion}

A maximum ion source temperature of $150{ }^{\circ} \mathrm{C}$ is available on the z-spray ESI source of the SYNAPT G2. This was insufficient to produce any meaningful ion current even for low molecular weight peptides such as angiotensin 1 (MW 1296) using 2,5-DHB matrix and the identical laser and configuration used in previous work [1-3]. Applying the fabricated temperature device (Graphical Abstract), multiply charged ions of peptides and pro-

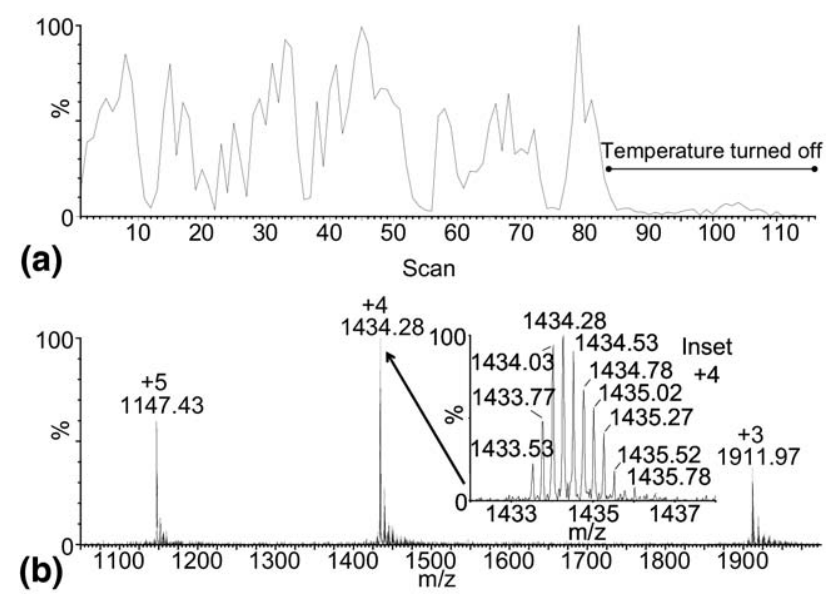

Figure 1. LSI-IMS-MS of bovine insulin (MW 5731): (a) The total ion current and (b) mass spectrum obtained using 2,5-DHB matrix incorporating the solvent-based sample preparation method. 
1)

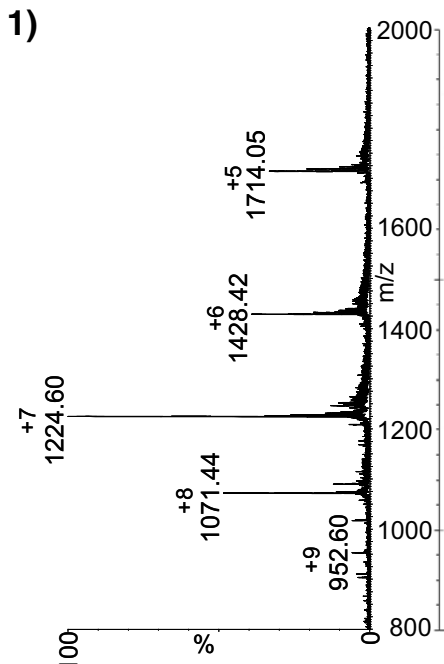

(a)

1)

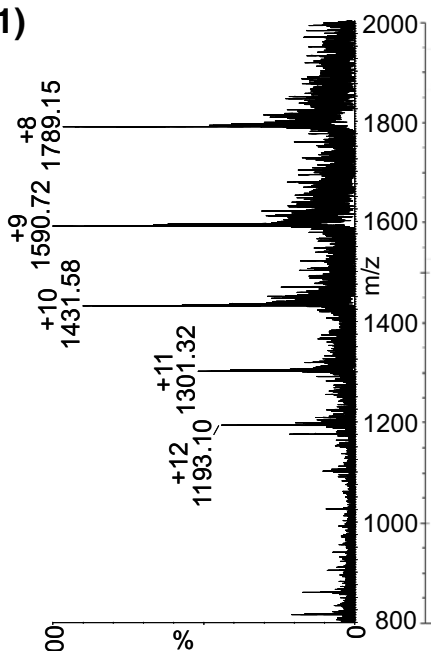

(b)

1)

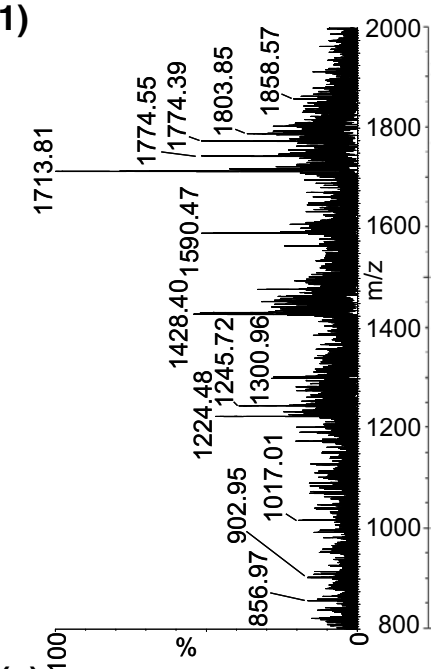

2)

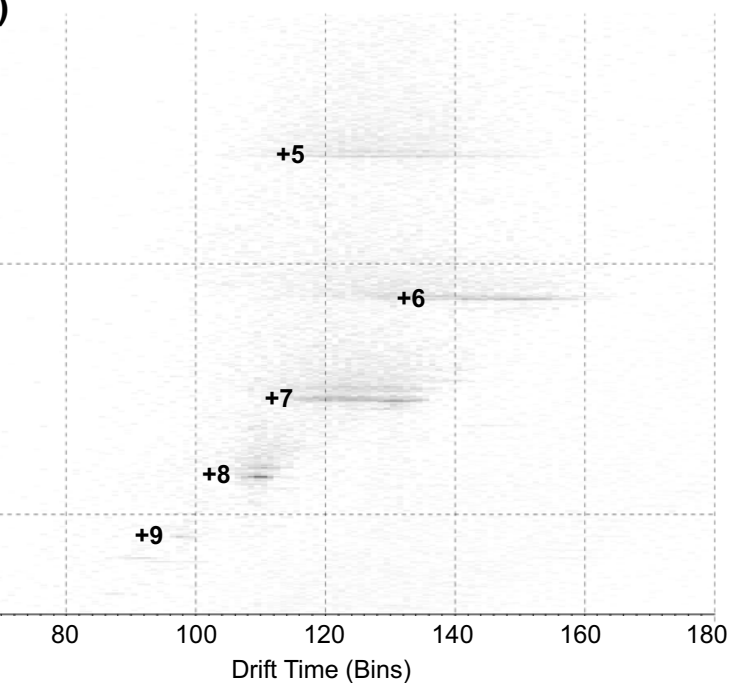

2)

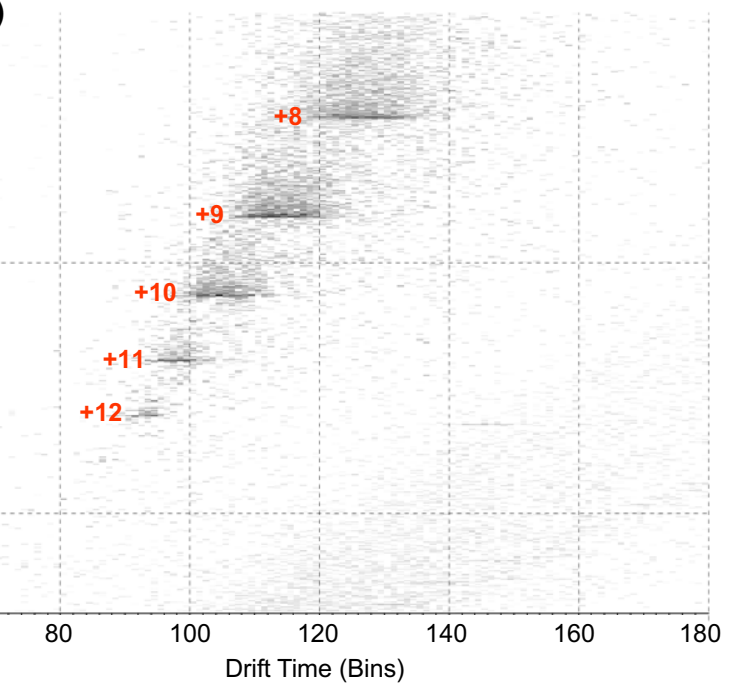

2)

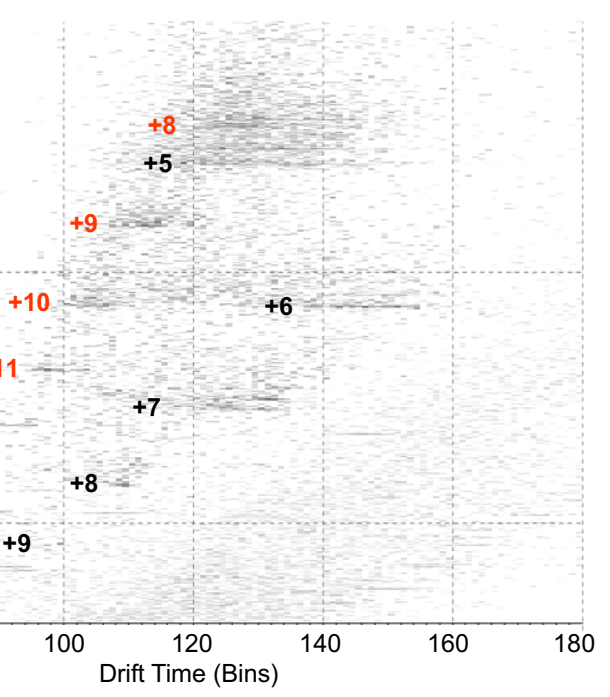

Figure 2. LSI-IMS-MS using 2,5-DHB matrix and the solvent-based sample preparation method of (a) ubiquitin (MW 8561), (b) lysozyme (MW 14,304), and (c) a mixture of ubiquitin and lysozyme. (1) Total mass spectra (as if no IMS dimension is employed) and (2) two-dimensional plots of drift time versus mass-to-charge ratio. 
teins are produced. A typical example is shown in Figure 1 using bovine insulin (MW 5731). The total ion current (Figure 1a) shows the continuous ion production when moving the sample stage over unablated areas of the sample showing an increase when heat (1-84 s) and a decrease when no heat (85-115 s) were applied. The charge state distribution (Figure $1 \mathrm{~b}$ ) is similar to that previously observed using the commercially available heated transfer capillary of the Thermo Fisher Scientific Ion Max ESI source of the Orbitrap Exactive and the LTQ mass spectrometers [2, 3]. Larger proteins such as ubiquitin (MW 8561) and lysozyme (MW 14,304) also produced multiply charged ions in good abundance (Figure 2.1) using the simple desolvation chamber. Myoglobin (MW 16,951), however, produced less abundant ions ranging from charge state +10 to +16 possibly suggesting a mass range limit for this particular desolvation device.

All experiments incorporated ion mobility separation. In ion mobility measurements, three-dimensional (3-D) information is acquired; $t_{d}, m / z$, and signal intensity. Frequently, the intensity of a signal is embedded in a 2-D plot of $t_{d}$ versus $m / z$ (Figure 2.2) and visualized by the use of a color-code. The 2-D IMS-MS plots of ubiquitin, lysozyme, and a mixture of ubiquitin and lysozyme are shown, respectively, in Figure 2.2. LSI ions of proteins fall into families depending upon their sizes and charge states as previously reported for biopolymers using ESI-IMS-MS [8]. Further, one can readily observe the elongated features indicating conformational differences within each charge state of each protein. Similar observations have been reported, especially for ubiquitin, using a homebuilt ESI-IMS-MS instrument [19-22].

Many features become noticeable in a 2-D plot. Without data extraction and time-intensive analyses, the pictorial snapshot [6] determines readily the presence of both proteins (Figure 2.2c). By selecting the specific part of the 2-D plot using the DriftScope, it is possible to extract $m / z$ and/or $t_{\mathrm{d}}$ information for any ion or inset region of interest. This removes most of the background interference, especially those at lower $m / z$. Most importantly, charge state families of different proteins can be extracted, providing the respective mass spectrum for each protein.

An example of extracting easily interpretable data from lower abundant ions is shown in Figure 3. The mass spectrum of a mixture of ubiquitin and lysozyme, purposely designed to have a high signal to noise ratio and to be difficult to interpret, is shown. It is not obvious from the mass spectrum that lysozyme is present, and it could easily be overlooked. Because the mobility dimension provides clean separation of the multiply charged ions of these proteins (Figure 2.2c), mass spectra can be extracted for both proteins free of the other. The extracted mass spectrum of lysozyme in the protein mixture is shown in Figure 3 and is similar to that of pure lysozyme (Figure $2.1 \mathrm{~b}$ ). The charge state

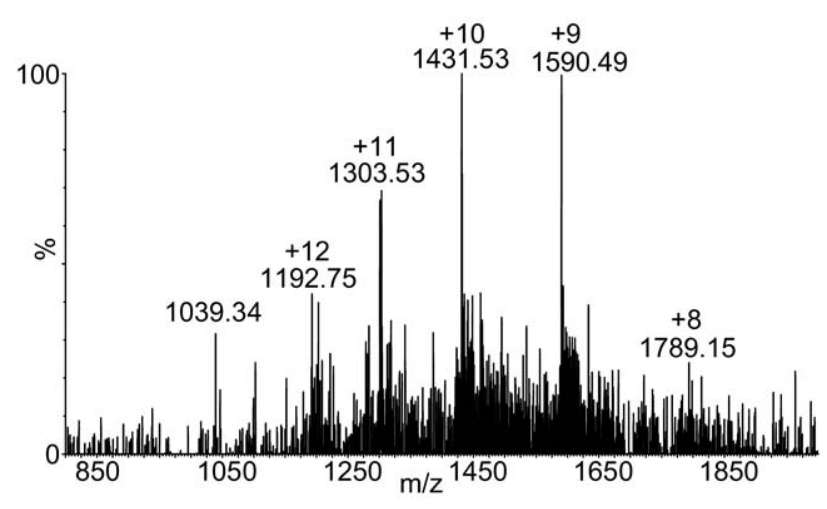

Figure 3. The LSI-IMS-MS mass spectrum of the extracted lysozyme component from the two-dimensional IMS-MS plot displayed in Figure 2.2c.

distributions are essentially identical with the most abundant ions having charged state +9 .

\section{Conclusion}

A simple desolvation device was constructed to convert highly charged matrix/analyte clusters formed by laser ablation of a matrix/protein mixture into multiply charged ions. The success of using a simple heated tube under AP conditions to produce multiply charged LSI ions lends further support to the proposal that the ionization mechanism in LSI is similar to ESI [3]. Further, this work suggests that a similar device retrofitted to any ESI source will produce highly charged ions using LSI. Thus, the ability to produce highly charged ions using a laser ablation process is readily available for advanced spectrometers with mass range limitations such as FT-ICR-MS and, as shown here, IMS-MS instruments. Solvent-free decongestion of protein mixtures using IMS-MS technology is especially promising for tissue imaging applications [1, 3, 14, 15]. Additionally, we are evaluating methods that lower the thermal requirement for desolvation and charge production.

\section{Acknowledgments}

Authors are grateful to Professor Charles N. McEwen (The University of the Sciences in Philadelphia) for providing the nitrogen laser, and Waters Corporation for their continuous support, especially Stephen Chadwick (Manchester, UK) and Alan Caporali (Milford, MA). The authors acknowledge funding from Wayne State University (Start-up Funds) and NSF CAREER 0955975.

\section{References}

1. Trimpin, S.; Herath, T. N.; Inutan, E. D.; Cernat, S. A.; Wager-Miller, J.; Mackie, K.; Walker, J. M. Field-free Transmission Geometry Atmospheric Pressure Matrix-Assisted Laser Desorption/Ionization for Rapid Analysis of Unadulterated Tissue Samples. Rapid Commun. Mass Spectrom. 2009, 23, 3023-3027.

2. Trimpin, S.; Inutan, E. D.; Herath, T. N.; McEwen, C. N. A MatrixAssisted Laser Desorption/Ionization Mass Spectrometry Method for Selectively Producing Either Singly or Multiply Charged Molecular Ions. Anal. Chem. 2010, 82, 11-15. 
3. Trimpin, S.; Inutan, E. D.; Herath, T. N.; McEwen, C. N. Laserspray Ionization - A New AP-MALDI Method for Producing Highly Charged Gas-Phase Ions of Peptides and Proteins Directly from Solid Solutions. Mol. Cell. Proteomics 2010, 9, 362-367.

4. Trimpin, S.; Plasencia, M. D.; Isailovic, D.; Clemmer, D. E. Resolving Oligomers from Fully Grown Polymers with IMS-MS. Anal. Chem. 2007, 79, 7965-7974.

5. Liu, X.; Valentine, S. J.; Plasencia, M. D.; Trimpin, S.; Naylor, S.; Clemmer, D. E. Mapping the Human Plasma Proteome by SCX-LCIMS-MS. J. Am. Soc. Mass Spectrom. 2007, 18, 1249-1264.

6. Trimpin, S.; Clemmer, D. E. Ion Mobility Spectrometry-Mass Spectrometry/ Mass Spectrometry for Assessing the Molecular Compositions of Complex Polymeric Systems. Anal. Chem. 2008, 80, 9073-9083.

7. Trimpin, S.; Tan, B.; Bohrer, B. C.; O’Dell, D. K.; Merenbloom, S. I.; Pazos, M. X.; Clemmer, D. E.; Walker, J. M. Profiling of Phospholipids and Related Lipid Structures using Multidimensional Ion Mobility Spectrometry-Mass Spectrometry. Int. J. Mass Spectrom. 2009, 287, 58-69.

8. Bohrer, B. C.; Merenbloom, S. I.; Koeniger, S. L.; Hilderbrand, A. E.; Clemmer, D. E. Biomolecule Analysis by Ion Mobility Spectrometry. Ann. Rev. Anal. Chem. 2008, 1, 293-327.

9. Kanu, A. B.; Hill, H. H. Ion Mobility Spectrometry Detection for Gas Chromatography. J. Chromatogr. A 2008, 1177, 12-27.

10. McLean, J. A.; Ruotolo, B. T.; Gillig, K. J.; Russell, D. H. Ion MobilityMass Spectrometry: A New Paradigm for Proteomics. Int. J. Mass Spectrom. 2005, 240, 301-315.

11. Kaur-Atwal, G.; O'Connor, G.; Aksenov, A. A.; Bocos-Bintintan, V.; Paul Thomas, C. L.; Creaser, C. S. Chemical Standards for Ion Mobility Spectrometry: A Review. Int. J. Ion Mobility Spectrom. 2009, 12, 1-14.

12. Trimpin, S.; Deinzer, M. L. Solvent-free MALDI-MS for the Analysis of $\beta$-Amyloid Peptides via the Mini-Ball Mill Approach: Qualitative and Quantitative Improvements. J. Am. Soc. Mass Spectrom. 2007, 18, 15331543.
13. Trimpin, S.; Herath, T. N.; Inutan, E. D.; Wager-Miller, J.; Kowalski, P.; Claude, E.; Walker, J. M.; Mackie, K. Automated Solvent-Free Matrix Deposition for Tissue Imaging by Mass Spectrometry. Anal. Chem. $\mathbf{2 0 1 0}$ 82, 359-367.

14. Trimpin, S.; Brizzard, B. Analysis of Insoluble Proteins. Biotechniques 2009, 46, 409-419.

15. Trimpin, S. A Perspective on Alternatives in MALDI - Total Solvent-free Analysis and Electron Transfer Dissociation of Highly Charged Ions. J. Mass Spectrom. 2010, doi:10.1002/jms1737.

16. Karas, M.; Hillenkamp, F. Laser Desorption Ionization of Proteins with Molecular Masses Exceeding 10,000 Daltons. Anal. Chem. 1988, 60, 2299-2301.

17. Vorm, O.; Roepstorff, P.; Mann, M. Improved Resolution and Very High Sensitivity in MALDI of Matrix Surfaces Made by Fast Evaporation. Anal. Chem. 1994, 66, 3281-3287.

18. Pringle, S. D.; Giles, K.; Wildgoose, J. L.; Williams, J. P.; Slade, S. E. Thalassinos, K.; Bateman, R. H.; Bowers, M. T.; Scrivens, J. H. An Investigation of the Mobility Separation of Some Peptide and Protein Ions Using a New Hybrid Quadrupole/Traveling Wave IMS/oa-TOF Instrument. Int. J. Mass Spectrom. 2007, 261, 1-12.

19. Koeniger, S. L.; Merenbloom, S. I.; Sevugarajan, S.; Clemmer, D. E. Transfer of Structural Elements from Compact to Extended States in Unsolvated Ubiquitin. J. Am. Chem. Soc. 2006, 128, 11713-11719.

20. Koeniger, S. L.; Merenbloom, S. I.; Clemmer, D. E. Evidence for Many Resolvable Structures within Conformation Types of Electrosprayed Ubiquitin Ions. J. Phys. Chem. B 2006, 110, 7017-7021.

21. Koeniger, S. L. Clemmer, D. E. Resolution and Structural Transitions of Elongated States of Ubiquitin. J. Am. Soc. Mass Spectrom. 2007, 18, 322-331.

22. Valentine, S. J.; Counterman, A. E.; Clemmer, D. E. Conformer-Dependent Proton-Transfer Reactions of Ubiquitin Ions. J. Am. Soc. Mass Spectrom. 1997, 8, 954-961. 